\title{
Frequency-specific Alterations of Functional Hub in Generalized Tonic-clonic Seizure*
}

\author{
Zhe Zhang ${ }^{1}$, Huiyan Cheng ${ }^{2}$, Xuhui Chen ${ }^{2}{ }^{\dagger}$, Chaolin $\mathrm{Ma}^{3}$ \\ ${ }^{1}$ Department of Electronics and Information Engineering, Lanzhou Institute of Technology, \\ 1 East Langongpin Road \\ Lanzhou, 730050, China \\ E-mail: zz.tute@163.com \\ ${ }^{2}$ Key of Internet of Things Lab, Xiamen University of Technology, \\ 600 Ligong Road \\ Xiamen, 361024, China \\ E-mail:xhchen@xmut.edu.cn \\ ${ }^{3}$ College of Life Sciences, Nanchang University, \\ 999 Xuefu road \\ Nanchang, 330031, China \\ E-mail: chaolin.ma@gmail.com
}

\begin{abstract}
Resting-state functional magnetic resonance imaging (rs-fMRI) has shown aberrant brain functional networks at a conventional low-frequency range $(0.01-0.08 \mathrm{~Hz})$ in individuals with generalized tonic-clonic seizure (GTCS). However, it is unclear how functional networks hubs change across different frequency bands in GTCS patients. In this study, we applied a data-driven method called complementary ensemble empirical mode decomposition (CEEMD) to decompose the whole brain blood oxygen level-dependent (BOLD) time series into five frequency-specific bands. Then, we compared alterations of frequency-specific functional hub across five different frequency bands in 21 GTCS patients to 22 normal control (NC) subjects. CTCS patients showed aberrant functional hubs predominately at some particular frequency bands and primary in the default mode network (DMN) and the somatomotor network (SMN). Furthermore, we found these hubs located in the DMN and the SMN at some specific bands $(0.015-0.025 \mathrm{~Hz}, 0.12-0.25 \mathrm{~Hz})$ were associated with disease severity. These findings highlight the role of frequency-specific functional hub, and may helpfully uncover abnormal neuronal activity in patients with GTCS at frequency-specific bands.
\end{abstract}

Keywords: Resting-state; GTCS; Hub; CEEMD; Frequency-specific.

\footnotetext{
* This work is financially supported by the National Health and Family Planning Commission Fund under grant WKJ-FJ-35, 3502Z20143031, 3502Z20133033, and the Scientific Innovation Foundation of Lanzhou Institute of Technology under grant 2014K-007.

${ }^{\dagger}$ Corresponding author.
} 


\section{Introduction}

Generalized tonic-clonic seizure(GTCS) is a common type of idiopathic generalized epilepsy (ICE) ${ }^{1}$. Generally, epileptic seizures are believed to affect a great number of brain regions and dramatically impair the patient' $s$ state of consciousness ${ }^{2}$. However, our understanding of the mechanisms of seizure initiation, maintenance, and termination during GTCS is still limited.

Resting-state functional magnetic resonance imaging (rs-fMRI) has been popularly applied to investigate spontaneous brain neural activity and has widely been performed to reveal intrinsic functional network abnormalities in various epilepsies ${ }^{3,4}$. To date, the majority of previous studies have predominately detected abnormal epileptic at a conventional lowfrequency range (0.01-0.08 Hz) because the lowfrequency range was thought to be linked to neuronal intrinsic fluctuations, which may ignore some meaningful information associated with frequencyspecific spontaneous blood oxygen level-dependent (BOLD) fluctuation ${ }^{5-8}$. Besides, neighboring frequency bands within the same neuronal network may compete or interact with each other, which means that the brain intrinsic resting-state functional networks are frequency-dependent ${ }^{9,10}$. However, few studies have concentrated on the changes in intrinsic activity that occurs across different frequency bands in patients with GTCS ${ }^{11}$. These studies usually investigated in resting-state functional connectivity by band passing the data into a priori defined frequency intervals.

The hypothesis suggests that brain functional network contains hubs which are important to process cortical information. Meanwhile, lesioning the hub thus result in severe cognitive impairment ${ }^{12}$. The existence and nature of hubs have become a major research target in brain functional networks ${ }^{13-}$ 15. Recently, a resting-state fMRI study has examined the impact of abnormalities in functional hubs in patients with GTCS $^{16}$. Importantly, the majority of research on hubs has been carried out under the assumption that resting-state brain function connectivity is consistent in time ${ }^{16-18}$. However, it is unclear how these hubs in brain functional networks change across different frequency bands in GTCS patients .

In the present study, we applied a data driven method called complementary ensemble empirical mode decomposition (CEEMD) to adaptively decompose the whole brain BOLD time series into several intrinsic frequency bands. Thereafter, the significant differences of hub in brain resting-state functional network between the two groups (GTCS and NC groups) at different frequency bands were identified and the severity of epileptic and cognitive function were explored. This study aim to uncover the neurophysiological significance of frequency-specific abnormal hub topological connectivity property in GTCS.

\section{Materials and Methods}

\subsection{Participants}

Patients with GTCS were recruited at Lanzhou General Hospital of Lanzhou Military Command, Lanzhou, China, between November 2011 and February 2013. We diagnosed patients according to the criteria of the International League Against Epilepsy (2001). A total of 23 patients with GTCS met these inclusion criteria. In the same period, we picked out 22 age- and sex-matched normal controls (NC) volunteers as a control group. No individuals suffer from neurological or psychiatric disorder and no gross abnormalities in brain MRI images. All the participants were given written informed consent. The local medical ethics committee at Lanzhou General Hospital of Lanzhou Military Command approved the study. Two groups handedness. At the end, 2 patients were excluded owing to excessive head motion parameters that exceeding 1.5 $\mathrm{mm}$ translation or $1.5^{\circ}$ in rotation. The demographic and clinical information of all the participants are detailed in Table 1.

Table 1. Demographic characteristics of the participants.

\begin{tabular}{|c|c|c|c|c|}
\hline & CTCS $(n=21)$ & Controls $(n=22)$ & $t$ value & $p$ value \\
\hline Sex (male/female) & $11 / 10$ & $11 / 11$ & - & - \\
\hline Age (years) & $18.58 \pm 6.49$ & $17.47 \pm 6.12$ & 0.449 & 0.675 \\
\hline Handedness(right/left) & $21 / 0$ & $22 / 0$ & - & - \\
\hline Years of education (years) & $8.53 \pm 2.15$ & $8.35 \pm 2.23$ & 0.201 & 0.842 \\
\hline NHS3 scores & $12.59 \pm 3.08$ & - & - & - \\
\hline
\end{tabular}



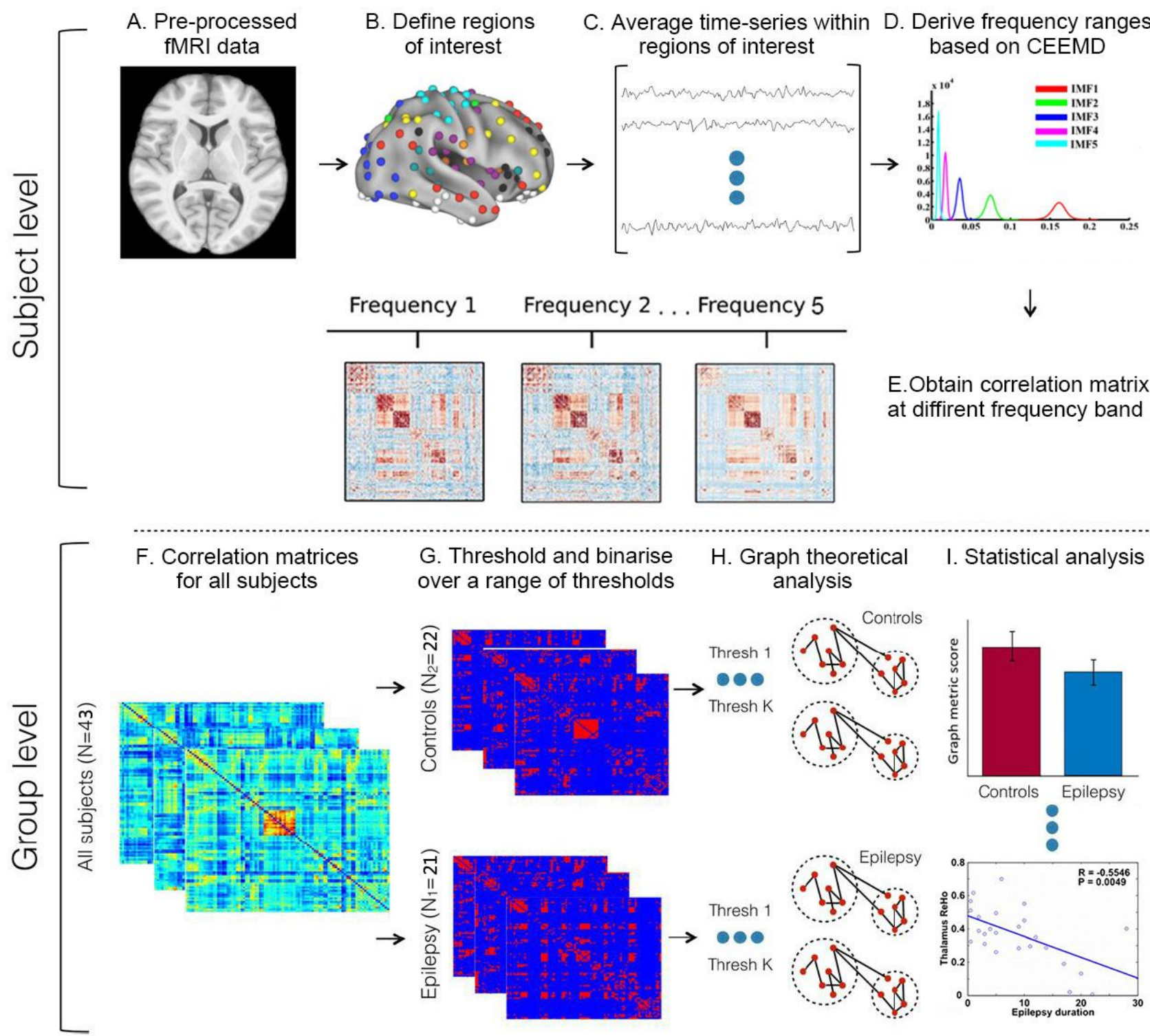

Fig. 1. A schematic overview of the analysis steps in this study.

\subsection{Data Acquisition}

All resting-state fMRI data were collected using a $3.0 \mathrm{~T}$ Siemens Trio scanner (Erlangen, Germany) with a 16 channel phased array head coil. During the data acquisition, participants were instructed to relax with their eyes closed, not think of anything in particular, keep their heads not moving in the scanner, and not fall asleep. The scan images were acquired using a GREEPI sequence (repetition time $=2000 \mathrm{~ms}$, echo time $=$
$30 \mathrm{~ms}$, slice thickness $=3.8 \mathrm{~mm}$, slice gap $=0.38 \mathrm{~mm}$, field of view $=240 \times 240 \mathrm{~mm}^{2}$, in-plane matrix $=64 \times$ 64, flip angle $=90^{\circ}$, and 34 slices covered the whole brain) to measure 200 functional volumes for each participant, resulting in a total scan time of 400 seconds.

\subsection{Data Preprocessing}

The functional data preprocessing was performed using DPARSF (http://www.restfmri.net). Firstly, the first 10 functional images volumes were removed to stabilize 
the signal and adapt to inherent scanner noise. Secondly, we corrected the functional images by slicetiming calibrating and realigning head motion. Next, functional images were normalized into standard threedimensional space at a resolution of $3 \times 3 \times 3 \mathrm{~mm}^{3}$ using the Montreal Neurological Institute (MNI) template. Moreover, several sources of spurious variances were discard by regression processes. Finally, any linear trends within the voxels were removed so as to discard signal drifts caused by scanner instability or other sources.

\subsection{Definition of Frequency-specific Band}

We adopted a data-driven method called complementary ensemble empirical mode decomposition (CEEMD) which was originally derived in Qian et $\mathrm{al}^{19}$ to separate the time series of each voxel into several intrinsic oscillation rhythms with distinct frequency bands. CEEMD is modified from a noise assisted ensemble empirical mode decomposition (EEMD), which produces an ensemble of data sets by adding different realizations of a white noise with finite amplitude $\varepsilon$ to the original data. The averaging impact of the assisted white noise $\varepsilon f$ decrease as:

$$
\varepsilon f=\varepsilon \cdot \operatorname{std}(y) / N E
$$

Where $\varepsilon$ is the input noise level, $y$ represents the input signal and $\mathrm{NE}$ is ensemble number.

Based on the CEEMD method, five distinct frequency bands were derived to cover a frequency range from 0 to $0.25 \mathrm{~Hz}$, with each interval range. This identified five frequency-specific bands including 0$0.016 \mathrm{~Hz}, 0.015-0.025 \mathrm{~Hz}, 0.025-0.055 \mathrm{~Hz}, 0.055-0.12$ $\mathrm{Hz}$, and $0.12-0.25 \mathrm{~Hz}$.

\subsection{Graph Theoretical Analysis}

Network Construction. In this study, we used the node template described by Power et $\mathrm{al}^{20}$. This template contains the subgraphs based on the known spatial structure of resting state fMRI network. We used a altered version where the 13 derived subgraphs were reduced to 5 subgraphs so that we could abtained hubs clearly. Throughout the paper, we used five subgraphs as follows: default mode network (DMN, 58 nodes), frontal-parietal network (FPN, 44 nodes), cinguloopercular network (CON, 32 nodes), somatomotor network (SMN, 35 nodes), visual-auditory network (VAN, 37 nodes). Fifty-one nodes were not assigned to any particular subgraph. Two hundred sixty-four $10 \mathrm{~mm}$ spherical nodes were defined along the cortex as well as in subcortical nuclei. Each node time series was extracted from each subject across five frequencies by averaging the BOLD signal intensity timecourse over all voxels in each of the 264 nodes. Pearson correlation coefficient was applied to estimate the correlation between any two nodes. We calculate the correlation among all of the 264 nodes, deducing 34848 correlation coefficients in every frequency band. Thus, we obtained a set of five $(264 \times 264)$ inter-regional Pearson correlation matrices for each subject. False discovery rate (FDR) correction was applied to regulate the expected FDR at the statistical significance threshold at 0.05 in individual level. Thus, five frequency dependent population based functional connectivity networks were constructed by capturing the underlying common connectivity pattern of the brain in each group.

Betweenness Centrality. We investigated the frequency dependency of node centrality by computing the node betweenness centrality (BC). BC is a sum of the shortest paths in the network that pass through a given node, which captures the effect of a node over information flow among other nodes in the network ${ }^{16}$. A high degree of $\mathrm{BC}$ is a hallmark property for nodes that are strong candidates to act as network hubs that are vital for the flow of information throughout the network $\mathrm{BC}$ of node $\mathrm{i}$ is defined as:

$$
B C=\frac{1}{(n-1)(n-2)} \sum_{\substack{h, j \subset N \\ h \neq j, h \neq i, j \neq i}} \frac{p_{h j}(i)}{p_{h j}}
$$

Where $p_{h j}$ is the number of shortest paths between $h$ and $j$, and $p_{h j}(i)$ is the number of shortest paths between $h$ and $j$ that pass through $\mathrm{i}$.

For the comparison between frequency-specific bands, each subjects $\mathrm{BC}$ were normalized over the frequency-specific band. Due to lack of a formal consensus regarding selection of thresholds, here a range of sparsity threshold $\left(S_{\min }(0.1) \leq\right.$ sparsity $\left.\leq 0.4\right)$ were selectedfor functional connectivity networks, where min s represented a minimum network sparsity where all nodes would become fully connected in the five frequency-specific bands brain networks. 

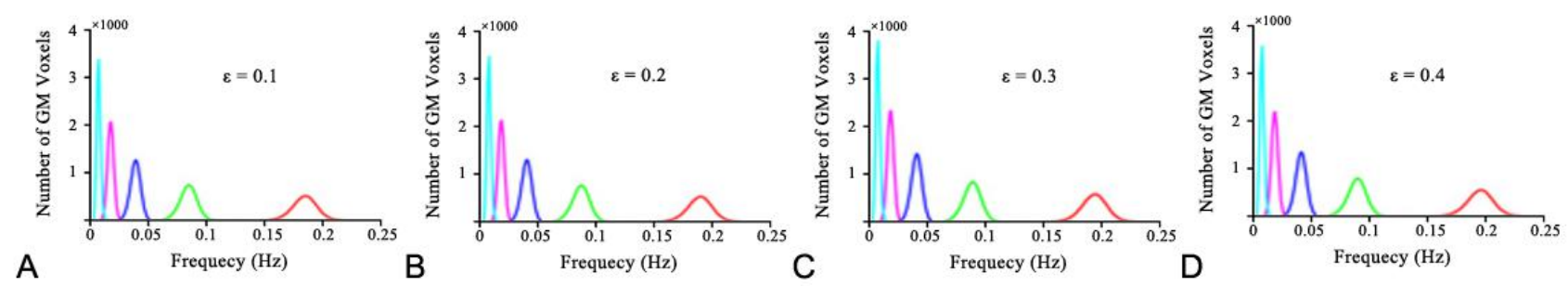

Fig. 2. Frequency distribution using CEEMD. From Fig. 2A to Fig. 2D, each figure represents the frequency distribution histogram determined from gray matter voxels in the whole brain across the entire group of subjects $(n=43)$, with an input noise level $\varepsilon$ of $0.1,0.2,0.3$ and 0.4 using CEEMD. The heights of the histograms represent the number of voxels whose histograms of frequency distribution equals to the frequency on the horizontal axis. The histograms of frequency distribution of the five frequency bands were colored by red, green, blue, magenta and cyan respectively. GM: gray matter.

\subsection{Statistical Analysis}

Two independent sample t-tests and Chi-squared tests were used to compare demographic performance $(\mathrm{p}<$ $0.05)$. For the $\mathrm{BC}$ measures used in this study, a subjectdependent value was obtained by averaging the measure over all graph nodes. Two-sample permutation t-tests assuming unequal variances between the two groups (epilepsy vs. controls) with 1000 non-parametric permutations were performed to quantify group differences at a specific threshold. For each permutation, group labels were randomly assigned to all subjects and the shuffled population was divided into two random groups. To further estimate the reliability of group effects, standardised effect sizes and the corresponding 95\% confidence intervals (CI) were calculated for all metrics. Finally, using SPSS 18.0 software (SPSS, Inc., Chicago, IL), correlative analysis between mean BC value and disease severity was then performed on the GTCS groups by extracting of the most significant different frequency between groups. A schematic overview of the analysis steps can see in Fig. 1

\section{Results}

\subsection{Frequency Distribution and Frequency Dependency of FC}

Fig. 2 showed the histograms to demonstrate the first five intrinsic mode functions of the voxels in the whole- brain gray matter at different input noise level $\varepsilon$ using the CEEMD method across all the subjects, and each of them represents the data of the whole-brain gray matter voxels within distinct frequency bands. The frequency band is the best to divide the resting-state fMRI signals. Finally, we derived five frequency-specific ranges including $0-0.016 \mathrm{~Hz}, 0.015-0.025 \mathrm{~Hz}, 0.025-0.055 \mathrm{~Hz}$, $0.055-0.12 \mathrm{~Hz}$, and $0.12-0.25 \mathrm{~Hz}$. Moreover, We performed a statistical comparison between the two groups over each of the frequency bands. In both the groups, the FC matrices of each frequency-specific band was presented in Fig. 3 to describe the inter-regional FC subtended by time series components. Across the two groups, the results indicated that the FC matrices continuously fluctuated at different frequency bands.

\subsection{Frequency-specific Alternating of Functional $\mathrm{Hub}$}

We have investigated whether the existence of functional hubs is consistent to across five frequency bands in both the groups. In order to derive functional hubs, the $\mathrm{BC}$ values for all nodes were computed in all brain functional networks for all five frequencies. The spatial layout of the frequency-dependent hubs is shown in Fig. 4A-B. The results suggested that the spatial distribution of functional hubs was not a constant arrangement, but rather re-configured in space that 

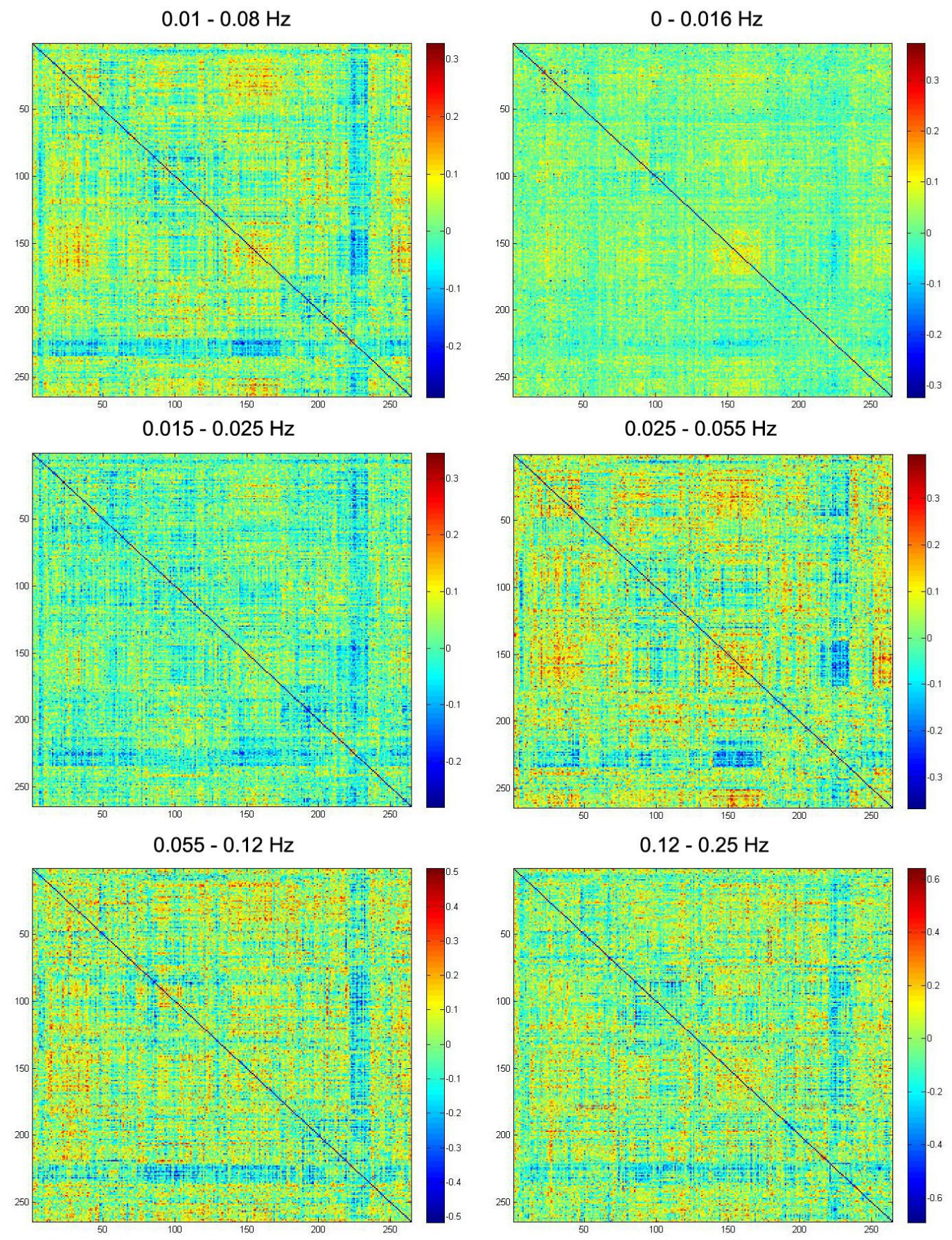

Fig. 3. Frequency-specific alternating of functional connectivity networks between-group. These correlation matrices denote the differences in functional connectivity networks for each specific frequency for both the groups $(264 \times 264$ correlation matrix, the number from 1 to 264 represents the corresponding ROI in Power' s template.), respectively. Hot color denote GTCS $>$ NC, cool color denote GTCS $<$ NC. 


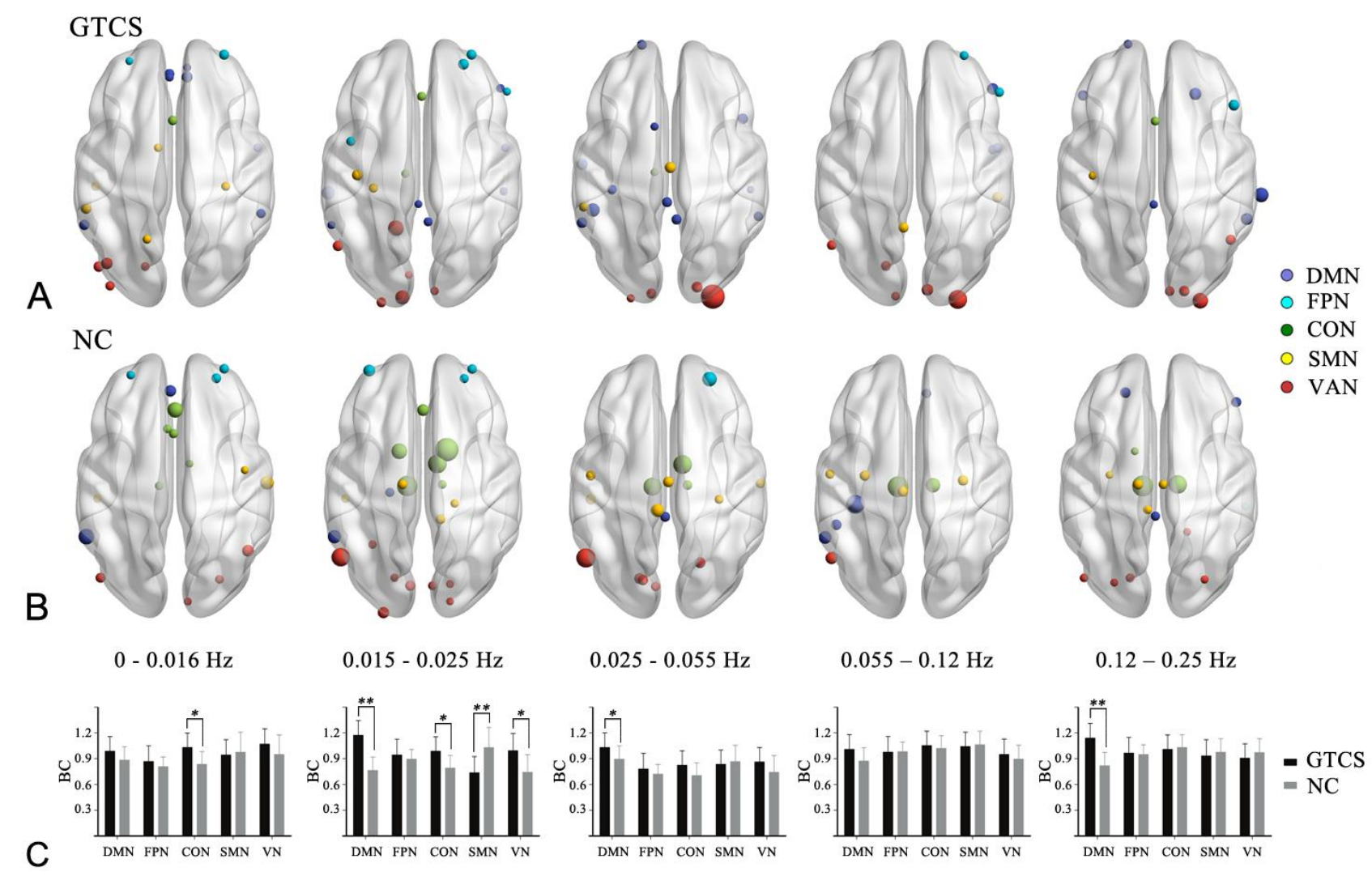

Fig. 4. Frequency-specific alternating of functional hub in within-group and between-group. Three dimensional rendering maps showed hub regions defined by BC in five frequency bands for the GTCS (Fig. 4A) group and the NC (Fig. 4B) group. The hub nodes shown in red, green, yellow,cyan and magenta color donate DMN, SMN, CON, FPN and VN subgraphs respectively as described by Power et $\mathrm{al}^{20}$. The size of the hub represents their $\mathrm{BC}$ values. The bar graph showed the mean $\mathrm{BC}$ values of subgraphs in both the groups with different frequency bands (Fig. 4C). The hubs are presented on three dimensional rendering maps using the BrainNet viewer (www.nitrc.org/projects/bnv/). DMN: Default mode network, FPN: Frontal parietal network, CON: Cingulo-opercular network, SMN: Somatomotor network, VAN: Visual-auditory network. $*$ : $<<0.05 ; * * \mathrm{p}<0.01$.

Table 2. The number of hubs for all five frequencies for each of the five subgraphs investigated.

\begin{tabular}{|c|c|c|c|c|c|c|c|c|c|c|}
\hline \multirow[b]{2}{*}{ Frequency bands } & \multicolumn{5}{|c|}{ GTCS } & \multicolumn{5}{|c|}{$\mathrm{NC}$} \\
\hline & $\mathrm{DMN}$ & FPN & $\mathrm{CON}$ & $\mathrm{SMN}$ & VAN & $\mathrm{DMN}$ & FPN & $\mathrm{CON}$ & SMN & VAN \\
\hline $0-0.016 \mathrm{~Hz}$ & 7 & 2 & 1 & 5 & 4 & 2 & 3 & 5 & 3 & 4 \\
\hline $0.015-0.025 \mathrm{~Hz}$ & 7 & 4 & 2 & 2 & 6 & 2 & 3 & 6 & 4 & 8 \\
\hline $0.025-0.055 \mathrm{~Hz}$ & 11 & 0 & 1 & 2 & 4 & 1 & 1 & 3 & 6 & 5 \\
\hline $0.055-0.12 \mathrm{~Hz}$ & 3 & 2 & 0 & 2 & 6 & 4 & 0 & 2 & 4 & 1 \\
\hline $0.12-0.25 \mathrm{~Hz}$ & 5 & 1 & 1 & 1 & 4 & 3 & 0 & 3 & 4 & 5 \\
\hline
\end{tabular}

depends on the underlying frequency range of the BOLD resting-state signal. To further investigate the heteroscedastic dependence on frequency of functional hubs and their difference between subgraphs, we computed the mean $\mathrm{BC}$ values for all subgraphs in each frequency specific brain network of the two groups as 
A

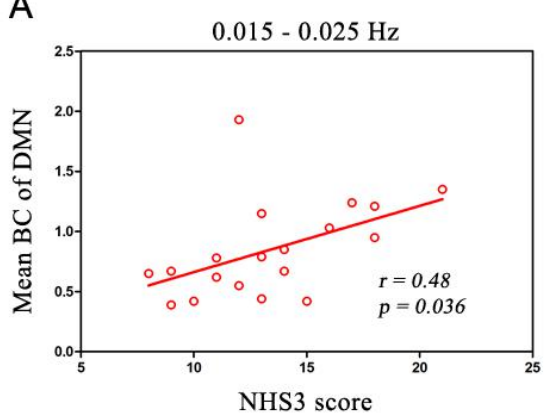

B

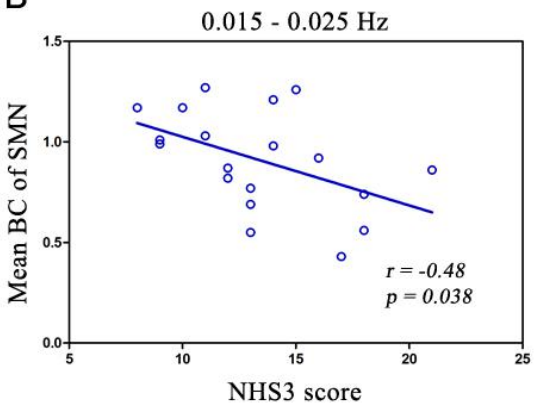

C

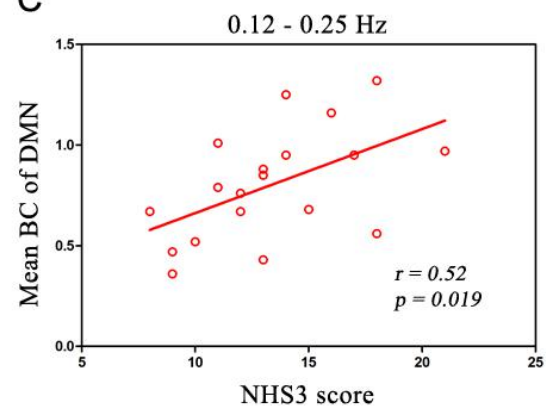

Fig. 5. Relationship between abnormal hub and disease severity. The NHS3 score of the GTCS patient was positively correlated with mean $\mathrm{BC}$ value in the $\mathrm{DMN}$ at $0.015-0.025 \mathrm{~Hz}$ (Fig. 5A) and $0.12-0.25 \mathrm{~Hz}$ (Fig. 5C), whereas negatively correlated with mean $\mathrm{BC}$ value in the $\mathrm{SMN}$ at $0.015-0.025 \mathrm{~Hz}$ (Fig. 5B).

shown in Fig. 4C. We found the significant differences of mean $\mathrm{BC}$ values mainly concentrated on the default mode network (DMN) and Somatomotor network (SMN). Particularly, to localize the specific frequency that exhibited alterations in hub in GTCS patient, we computed mean $\mathrm{BC}$ values of each subgraph in the five frequency bands. The results showed the GTCS patients had significantly higher $\mathrm{BC}$ at two specific frequencies $(0.015-0.025$ and $0.12-0.25 \mathrm{~Hz})$ in DMN and lower $\mathrm{BC}$ at one specific frequency $(0.015-0.025$ $\mathrm{Hz})$ in SMN.

Moreover, we have counted the number of hubs in each of the subgraphs for all five frequencies in both the groups as shown in Table 2. The number of hubs in several subgraphs are continuously changing in some specific frequencies. Interestingly, compared to $\mathrm{NC}$ group, the highest number of hubs in the FPN is found at $0.025-0.055 \mathrm{~Hz}$, at $0.05-0.12 \mathrm{~Hz}$ for the DMN subgraph, while the lowest number of hubs in the CON is found at $0.025-0.055 \mathrm{~Hz}$, at $0-0.016 \mathrm{~Hz}$ and at $0.015-0.025 \mathrm{~Hz}$ for the SMN subgraph in GTCS group. In general, our results suggested that altering functional hubs in GTCS happened at specific frequencies.

\subsection{Relationship Between Abnormal Hub and Disease Severity}

Clinical relevance analysis indicated that a significant correlation in DMN and SMN at some frequencyspecific bands was found. The mean $\mathrm{BC}$ values of the node located in posterior portion of the DMN are positively correlated with the NHS3 scores in patients with CTCS at the $0.015-0.025 \mathrm{~Hz}$ band (Fig. 5A, r =
$0.48, \mathrm{P}=0.036$ ) and at the $0.12-0.25 \mathrm{~Hz}$ band (Fig. $5 \mathrm{C}$, $\mathrm{r}=0.52, \mathrm{P}=0.019)$, whereas negatively correlated with mean $\mathrm{BC}$ value in the SMN at $0.015-0.025 \mathrm{~Hz}$ (Fig. $5 \mathrm{~B})$. We found that for the remaining frequency bands, there was no significant correlation between the BC values and the disease duration, onset age, or seizure frequency.

\section{Discussion}

This study aims to investigate frequency-specific alterations of functional hub in patients with CTCS. The principal finding was that CTCS patients showed aberrant functional hubs predominately at some particular frequency bands and primary in the DMN. Additionally, we found that abnormal brain functional hubs in the DMN at a specific higher frequency band between two groups were associated with the severity of GTCS. In general, these findings highlight a new view of frequency-specific hub in resting-state functional network, and may be helpful to uncover abnormal functional network in GTCS at specific frequency bands.

Previous studies have indicated that some brain rhythms organized many brain oscillations in order to support the complex brain activities within clear frequency bands. And in the same or different brain areas, these rhythms could temporally coexist. Due to specific properties and physiological functions, these rhythms may also interact with each other ${ }^{21,22}$. CEEMD which is a data driven method was verified an efficient method in the aspect of separating the intrinsic coexisting rhythms that are in distinct frequency bands 
because this method has the capability to eliminate mode-mixing effects ${ }^{19}$. In the current study, we applied the CEEMD to separate the inherent brain oscillations which were embedded in BOLD signals and the results have suggested that frequency characteristics of $\mathrm{FC}$ are strictly related with the corresponding inter-regional physical distance in both the groups.

We surveyed band-specific changes of brain functional hub in full frequency bands $(0-0.25 \mathrm{~Hz})$ in GTCS. It is not surprising that we observed continuous hub changes across the five frequency bands, and the changes primarily appeared in the DMN and in patients with GTCS, which is consistent with the previous findings ${ }^{11,16,23}$. We have also observed the amplitude of low-frequency fluctuation (ALFF) and regional homogeneity (ReHo) changes in resting-state brain intrinsic fluctuations at different frequency bands in the GTCS patients ${ }^{11,23}$. The frequency-specific strategy has been thought to be advantage able because it can provide more overall information about changed BOLD signals in the patients with GTCS. Interestingly, we found that the decreased $\mathrm{BC}$ mainly in the lower frequency bands $(0.015-0.025 \mathrm{~Hz})$, while the increased $\mathrm{BC}$ predominately in the higher frequency bands $(0.12$ $0.25 \mathrm{~Hz}$ ). This indicates that frequency-specific functional DMN impairment in patients with GTCS is not constant in full frequency bands. Fortunately, this finding has first been reported in a human functional neuroimaging study of epilepsy. It is normally confirmed that the DMN is strictly correlated to human brain consciousness ${ }^{24}$. GTCS is a remarkable type of convulsive seizure, after which consciousness is deeply damaged. Cerebral blood flow studies are beneficial for the reason of employing an injection of radio tracer during a seizure attempt. Therefore, researchers can identify abnormal activity that possibly contributes to the ictal impairment of consciousness ${ }^{25}$. Our findings suggest that aberrant functional hubs occurs in the conscious-related network in patients with GTCS at some specific frequency. Although we don't know well the correspondence between the BOLD frequency bands investigated here and EEG rhythms, it seems probable that the different frequency band of BOLD fluctuations may play a particular role, indicating that frequencyspecific selection is useful for clinical therapies for epilepsy.

The findings suggest that the hubs located in the DMN at a higher frequency band $(0.12-0.25 \mathrm{~Hz})$ showed increasing BC as the NHS3 scores in patients with GTCS increased. This altered functional hubs in the DMN may reflect the long-term injurious effects of epileptic activity, suggesting particular defective inhibition of the DMN in GTCS. This finding is consistent with the previous functional connectivity studies $^{17,26}$. Moreover, the frequency-specific reversal of functional hubs across 5 frequency bands located in the DMN indicates that the DMN is modulated by frequency in the GTCS patients. These findings uncover the DMN may encompass frequency-specific hierarchical functions, corresponding with the frequency-specific local synchronization of BOLD fluctuations. In general, these results illustrate that the DMN in different frequency bands is correlated with disease, which deepens our understanding of frequencyspecific pathology in patients with GTCS.

However, the study still has certain limitations. First, this was a statistic study between two groups with a relatively small sample size, which may contribute to some incorrect results. Second, we aim to study the spontaneous brain activities, but the thoughts of the participants during imaging may be the interference factors. Finally, the participants still have light head movements despite being demanded not to move their heads and being asked to close their eyes during imaging. However, we inspected each image and patients with head movements greater than $1.5 \mathrm{~mm}$ translation or $1.5^{\circ}$ in rotation were excluded.Taking these limitations into consideration, future studies should be well designed and should include larger numbers of participants.

Despite the limitations, this study first observed that the aberrant hub distribution of brain functional networks are frequency-dependent in GTCS patients. Abnormal functional hubs at a specific high-frequency in the DMN can serve as potential clinical biomarker for GTCS.

\section{References}

1. B.S. Chang, and D.H. Lowenstein, 'Practice Parameter: Antiepileptic Drug Prophylaxis in Severe Traumatic Brain Injury: Report of the Quality Standards Subcommittee of the American Academy of Neurology', Neurology, 60 (2003), 10-16.

2. H. Stefan, and F. H. Lopes da Silva, 'Epileptic Neuronal Networks: Methods of Identification and Clinical Relevance', Front Neurol, 4 (2013), 8. 
3. Marielle C. G. Vlooswijk, Jacobus F. A. Jansen, Marc Cftm de Krom, H. J. Marian Majoie, Paul A. M. Hofman, Walter H. Backes, and Albert P. Aldenkamp, 'Functional Mri in Chronic Epilepsy: Associations with Cognitive Impairment', The Lancet Neurology, 9 (2010), 1018-27.

4. W. Liao, Z. Zhang, D. Mantini, Q. Xu, G. J. Ji, H. Zhang, J. Wang, Z. Wang, G. Chen, L. Tian, Q. Jiao, Y. F. Zang, and G. Lu, 'Dynamical Intrinsic Functional Architecture of the Brain During Absence Seizures', Brain Struct Funct, 219 (2014), 2001-15.

5. D. Zhang and M. E. Raichle, 'Disease and the brain's dark energy', Nat Rev Neurology, 6 (2010), 15 - 28.

6. J. B. Kim, S. I. Suh, W. K. Seo, K. Oh, S. B. Koh, and J. H. Kim, 'Altered Thalamocortical Functional Connectivity in Idiopathic Generalized Epilepsy', Epilepsia, 55 (2014), 592-600.

7. U. Seneviratne, M. Cook, and W. D'Souza, 'Focal Abnormalities in Idiopathic Generalized Epilepsy: A Critical Review of the Literature', Epilepsia, 55 (2014), 1157-69.

8. Z. Zhang, Q. Xu, W. Liao, Z. Wang, Q. Li, F. Yang, Z. Zhang, Y. Liu, and G. Lu, 'Pathological Uncoupling between Amplitude and Connectivity of Brain Fluctuations in Epilepsy', Hum Brain Mapp, 36 (2015), 2756-66.

9. A. K. Engel, P. Fries and W, Singer, 'Dynamic predictions:oscillations and synchrony in top-down processing', Nat Rev Neurosci, 2 (2011), 704 - 716.

10. W. H. Thompson, and P. Fransson, 'The Frequency Dimension of Fmri Dynamic Connectivity: Network Connectivity, Functional Hubs and Integration in the Resting Brain', Neuroimage, 121 (2015), 227-42.

11. J. Wang, Z. Zhang, G. J. Ji, Q. Xu, Y. Huang, Z. Wang, Q. Jiao, F. Yang, Y. F. Zang, W. Liao, and G. Lu, 'Frequency-Specific Alterations of Local Synchronization in Idiopathic Generalized Epilepsy', Medicine (Baltimore), 94 (2015), e1374.

12. X. N. Zuo, R. Ehmke, M. Mennes, D. Imperati, F. X. Castellanos, O. Sporns, and M. P. Milham, 'Network Centrality in the Human Functional Connectome', Cereb Cortex, 22 (2012), 1862-75.

13. K. Gopinath, V. Krishnamurthy, R. Cabanban, and B. A. Crosson, 'Hubs of Anticorrelation in High-Resolution Resting-State Functional Connectivity Network Architecture', Brain Connect, 5 (2015), 267-75.

14. Z. Zhang, W. Liao, H. Chen, D. Mantini, J. R. Ding, Q. $\mathrm{Xu}, \mathrm{Z}$. Wang, C. Yuan, G. Chen, Q. Jiao, and G. Lu, 'Altered Functional-Structural Coupling of Large-Scale Brain Networks in Idiopathic Generalized Epilepsy', Brain, 134 (2011), 2912-28.
15. M. Pedersen, A. H. Omidvarnia, J. M. Walz, and G. D. Jackson, 'Increased Segregation of Brain Networks in Focal Epilepsy: An Fmri Graph Theory Finding', Neuroimage Clin, 8 (2015), 536-42.

16. M. Pedersen, A. H. Omidvarnia, J. M. Walz, and G. D. Jackson, 'Increased Segregation of Brain Networks in Focal Epilepsy: An Fmri Graph Theory Finding', Neuroimage Clin, 8 (2015), 536-42.

17. H. L. Wei, J. An, L. L. Zeng, H. Shen, S. J. Qiu, and D. W. Hu, 'Altered Functional Connectivity among Default, Attention, and Control Networks in Idiopathic Generalized Epilepsy', Epilepsy Behav, 46 (2015), 11825.

18. J. D. Power, B. L. Schlaggar, C. N. Lessov-Schlaggar, and S. E. Petersen, 'Evidence for Hubs in Human Functional Brain Networks', Neuron, 79 (2013), 798-813.

19. L. Qian, Y. Zhang, L. Zheng, Y. Shang, J. H. Gao, and Y. Liu, 'Frequency Dependent Topological Patterns of Resting-State Brain Networks', PLoS One, 10 (2015), e0124681.

20. J. D. Power, A. L. Cohen, S. M. Nelson, G. S. Wig, K. A. Barnes, J. A. Church, A. C. Vogel, T. O. Laumann, F. M. Miezin, B. L. Schlaggar, and S. E. Petersen, 'Functional Network Organization of the Human Brain', Neuron, 72 (2011), 665-78.

21. G. Buzsa, and A. Draguhn, 'Neuronal Oscillations in Cortical Networks', Science, 304 (2004), 1926-29.

22. Siegel M, Donner TH, and Engel AK., 'Spectral fingerprints of large-scale neuronal interactions', Nat Rev Neurosci, 13 (2012), 121 - 134.

23. Z. Wang, Z. Zhang, W. Liao, Q. Xu, J. Zhang, W. Lu, Q. Jiao, G. Chen, J. Feng, and G. Lu, 'Frequency-Dependent Amplitude Alterations of Resting-State Spontaneous Fluctuations in Idiopathic Generalized Epilepsy', Epilepsy Res, 108 (2014), 853-60.

24. H. Blumenfeld, 'Epilepsy and the consciousness system: transient vegetative state?', Neurol Clin, 29 (2011), 801823.

25. S. Seri, D. Brazzo, N. J. Thai, and A. Cerquiglini, 'Brain Mechanisms of Altered Consciousness in Generalised Seizures', Behav Neurol, 24 (2011), 43-6.

26. M. Pedersen, E. K. Curwood, J. S. Archer, D. F. Abbott, and G. D. Jackson, 'Brain Regions with Abnormal Network Properties in Severe Epilepsy of LennoxGastaut Phenotype: Multivariate Analysis of Task-Free Fmri', Epilepsia, 56 (2015), 1767-73. 